\title{
HOMOMORPHISMS OF FILTERED MODULES
}

\author{
BY \\ ALEX HELLER
}

1. Introduction. The following simple problem appears to be without a known solution. Let $A^{\prime}, A^{\prime \prime}, B^{\prime}, B^{\prime \prime}$ be modules and suppose $\alpha \in \operatorname{Ext}^{1}\left(A^{\prime \prime}, A^{\prime}\right), \beta \in \operatorname{Ext}^{1}\left(B^{\prime \prime}, B^{\prime}\right)$. Then $\alpha$ and $\beta$ determine extensions $A$ of $A^{\prime \prime}$ by $A^{\prime}$ and $B$ of $B^{\prime \prime}$ by $B^{\prime}$. How may the group $\operatorname{Hom}(A, B)$ be expressed in terms of the four modules and two extension classes?

We give here a partial solution of this problem. $\operatorname{Hom}(A, B)$ is seen to have a canonical filtration; we compute the associated graded group. Our failure to compute $\operatorname{Hom}(A, B)$ itse!f is to be thought of as analogous to the failure of the Künneth theorem to compute canonically the homology of a product of chain complexes (cf. [2]).

This partial solution is a special case of a solution of a more general problem. If $A$ and $B$ are filtered modules then how may $\operatorname{Hom}(A, B)$, or rather, its associated graded group, be computed in terms of the associated graded modules $A^{\prime \prime}$ and $B$ " of $A$ and $B$, together with the "extension classes" needed to reconstruct $A$ and $B$ from these graded modules? Indeed a similar question arises with respect to all the groups $\operatorname{Ext}^{q}(A, B)$.

We shall see that associated with such data there is a spectral sequence whose term $E^{1}$ is just $\operatorname{Ext}\left(A^{\prime \prime}, B^{\prime \prime}\right)$, and whose term $E^{\infty}$ is associated with a filtration of $\operatorname{Ext}(A, B)$. The derivation $d^{1}$ is easily computed. No attempt is made to compute the higher derivations in the general case. However, in the special case with which we began, $d^{2}$, which is the only other nonvanishing one, is explicitly computed as a secondary operation on $\operatorname{Ext}\left(A^{\prime \prime}, B^{\prime \prime}\right)$.

Our attention is restricted here to modules with finite filtrations. It is not particularly difficult to generalize the results to arbitrary increasing filtrations of modules. However the results stated here go over without any essential change to filtrations in arbitrary exact categories, or indeed in additive categories with abelian structures [1]. This would not be true of infinite filtrations. The restriction is adopted for this reason.

It is proposed to use the information obtained here in the study of universal coefficient and Künneth theorems for chain complexes of modules. The present study is however completely independent of such application. Since it throws some light on the problem originally mentioned it seems appropriate to present it separately.

Received by the editors April 20, 1962. 
2. The category of filtered modules. We shall for the whole of this paper suppose that there is, somewhere in the background, a ring, that all modules considered are left modules over this ring, and that all homomorphisms of modules are operator homomorphisms with respect to this ring.

By a graded module we shall mean a sequence of modules (indexed by negative as well as positive integers); we refrain from identifying it with the sum of its terms. For filtered modules we adopt the convenient if somewhat unconventional definition of [3]; a filtered module $\mathbf{X}$, or filtration $\mathbf{X}$ is a short exact sequence

$$
\mathbf{X}=\left(0 \rightarrow X \stackrel{x}{\rightarrow} X \stackrel{x^{\prime \prime}}{\rightarrow} X^{\prime \prime} \rightarrow 0\right)
$$

of graded modules, with $x, x^{\prime \prime}$ homogeneous of degrees 1,0 . The graded module $X^{\prime \prime}$ is of course the associated graded module of $\mathbf{X}$. The filtration is increasing if $X_{n}=0$ for some $m$ (and thus all $k \leqq m$ ) and decreasing if for all $k$ greater than some $n$ the modules $X_{k}^{\prime \prime}$ are 0 . If it is both it is a finite filtration.

In this paper we restrict ourselves to finite filtrations. For such a filtration $\mathbf{X}$ (and indeed for any decreasing filtrations) we have, for sufficiently large $k$, $X_{k} \approx X_{k+1} \approx \cdots$; we denote the common value by $X_{\infty}$ and say that $\mathbf{X}$ is a filtration of $X_{\infty}$.

If $\mathbf{X}$ and $\mathbf{Y}=\left(0 \rightarrow Y \stackrel{y}{\rightarrow} Y \stackrel{\nu^{\prime \prime}}{\rightarrow} Y^{\prime \prime} \rightarrow 0\right)$ are filtrations we define the graded abelian group $\operatorname{Hom}(\mathbf{X}, \mathbf{Y})$ by letting $\operatorname{Hom}_{q}(\mathbf{X}, \mathbf{Y})$ be the set of pairs $\left(f, f^{\prime \prime}\right)$ of maps, each homogeneous of degree $q$, such that

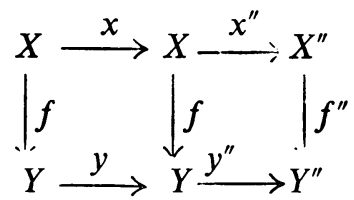

commutes. With this convention the filtered modules clearly become the objects of a graded additive category $\mathscr{F}$. This category is never exact, but it has a natural abelian structure (cf. [1]) given by the convention that $\left(f, f^{\prime \prime}\right)$ is a proper monomorphism (epimorphism) whenever both $f$ and $f^{\prime \prime}$ are monomorphisms (epimorphisms). We shall always consider $\mathscr{F}$ with this structure, which is perhaps more neatly characterized by the following lemma:

LEMMA 2.1. $\left(f, f^{\prime \prime}\right): \mathbf{X} \rightarrow \mathbf{Y}$ is a proper monomorphism (epimorphism, isomorphism) if and only if $f^{\prime \prime}: X^{\prime \prime} \rightarrow Y^{\prime \prime}$ is a monomorphism (epimorphism, isomorphism).

This follows immediately from the finiteness of the filtrations.

We denote by $S^{\prime \prime}$ the functor $S^{\prime \prime} \mathbf{X}=X^{\prime \prime}, S^{\prime \prime}\left(f, f^{\prime \prime}\right)=f^{\prime \prime}$, which maps $\mathscr{F}$ onto the category of finitely graded modules. It is, by 2.1 , exact. If $X^{\prime \prime}$ is a finitely graded module an extension of $X^{\prime \prime}$ is a filtration $\mathbf{X} \in \mathscr{F}$ with $S^{\prime \prime} \mathbf{X}=X^{\prime \prime}$. If $\mathbf{X}$ and $\mathbf{Y}$ are extensions of $X^{\prime \prime}$ we say that they are equivalent if there is an $f: X \rightarrow Y$ with $S^{\prime \prime} f=1: X^{\prime \prime}$. The set of equivalence classes of such extensions is denoted by $\mathbb{E} X$. If $\mathbf{X}$ is such an extension it determines an extension-class $\mathbf{e X} \in \mathfrak{E} X^{\prime \prime}$. 
We shall also want to use the above notions for graded modules. A filtered graded module $\mathbf{X}$ is a short exact sequence $0 \rightarrow X \stackrel{x}{\rightarrow} X \stackrel{x^{\prime \prime}}{\rightarrow} X^{\prime \prime} \rightarrow 0$ of bigraded modules with maps $x$ of bidegree $(1,0)$ and $x^{\prime \prime}$ of bidegree $(0,0)$; we refer to the two degrees as filtering and auxiliary degrees. The finiteness conditions are of course confined to the filtering degree. We content ourselves with asserting them in the strongest possible form: for some $m$, all $X_{i m}=0$, and for some $n$ and all $k>n$, all $X_{i k}^{\prime \prime}=0$. More generally, we may even discuss filtered multigraded modules, subject to the same conditions, which we describe by saying that the finiteness condition holds with respect to the filtering degree uniformly in the other degrees.

3. Splittings, projectives and the filtration of $\operatorname{Hom}(X, Y)$. If $\boldsymbol{X}$ is a filtered module, a left-splitting of $\mathbf{X}$ is a map $\xi: X \rightarrow X$ homogeneous of degree -1 such that $\xi x=1: X$. The set of these (which is often empty) we denote by $\Psi \mathbf{X}$. A right-splitting of $\mathbf{X}$ is a map $\xi^{\prime \prime}: X^{\prime \prime} \rightarrow X$, of degree 0 , with $x^{\prime \prime} \xi^{\prime \prime}=1: X^{\prime \prime}$; the set of these is $\Psi^{*} \mathbf{X}$. A bijective correspondence $\xi \rightarrow \xi^{*}$ of $\Psi \mathbf{X}$ with $\Psi^{*} \mathbf{X}$ is given by the condition $\xi \xi^{*}=0$, which implies that $\left(x, \xi, \xi^{*}, x^{\prime \prime}\right)$ is a direct sum decomposition of $X$ as a sum of $X$ and $X^{\prime \prime}$, and in particular that $x \xi+\xi^{*} x^{\prime \prime}=1: X$.

LEMMA 3.1. If $\xi \in \Psi \mathbf{X}$ then $1: X=\sum_{n=0}^{\infty} x^{n} \xi^{*} x^{\prime \prime} \xi^{n}$.

Observe first that the sum on the right is in fact finite on each $X_{q}$. Now rewriting $\xi^{*} x^{\prime \prime}$ as $1: X-x \xi$ we have

$$
\sum_{n=0}^{\infty} x^{n} \xi^{*} x^{n} \xi^{n}=\sum_{n=0}^{\infty} x^{n} \xi^{n}-\sum_{n=1}^{\infty} x^{n} \xi^{n}
$$

which is of course sufficient.

Corollary 3.2. If also $\eta \in \Psi \mathbf{Y}$ and $\left(f, f^{\prime \prime}\right): \mathbf{X} \rightarrow \mathbf{Y}$ then

$$
f=\sum_{n=0}^{\infty} \sum_{k=0}^{n} y^{n} \eta^{*} y^{\prime \prime} \eta^{n} f x^{k} \xi^{*} x^{\prime \prime} \xi^{k}
$$

We need only observe, in order to see this, that terms with $k>n$ vanish. It is worth remarking that $y^{\prime \prime} f \xi^{*}=f^{\prime \prime}$ so that the term with $n=k=0$ is $y^{*} f^{\prime \prime} x^{\prime \prime}$.

Now for any $\mathbf{X}, \mathbf{Y} \in \mathscr{F}$ we have an exact sequence

$$
0 \rightarrow \operatorname{Hom}(\mathbf{X}, \mathbf{Y}) \stackrel{h}{\rightarrow} \operatorname{Hom}(\mathbf{X}, \mathbf{Y}) \stackrel{\tilde{h}}{\rightarrow} \operatorname{Hom}\left(X^{\prime \prime}, Y^{\prime \prime}\right)
$$

given by $h\left(f, f^{\prime \prime}\right)=(y f, 0)=(f x, 0)$ and $\tilde{h}\left(f, f^{\prime \prime}\right)=f^{\prime \prime}$. Here $h$ is homogeneous of degree 1 and $\tilde{h}$ of degree 0 . Thus $\operatorname{Hom}(\mathbf{X}, \mathbf{Y})$ is in a natural way a filtered group or, more precisely, there is a filtered group

$$
0 \rightarrow \operatorname{Hom}(\mathbf{X}, \mathbf{Y}) \stackrel{h}{\rightarrow} \operatorname{Hom}(\mathbf{X}, \mathbf{Y}) \stackrel{h^{\prime \prime}}{\rightarrow} \operatorname{Hom}^{\prime \prime}(\mathbf{X}, \mathbf{Y}) \rightarrow 0
$$

whose associated graded group $\operatorname{Hom}^{\prime \prime}(\mathbf{X} \mathbf{Y})$ is a subgroup of $\operatorname{Hom}\left(X^{\prime \prime}, Y^{\prime \prime}\right)$. The finiteness conditions clearly imply that $\operatorname{Hom}_{\infty}(\mathbf{X}, \mathbf{Y}) \approx \operatorname{Hom}\left(X_{\infty}, Y_{\infty}\right)$, so that $\left(3.3^{\prime}\right)$ is a filtration of $\operatorname{Hom}\left(X_{\infty}, Y_{\infty}\right)$. One of our principal aims here is the computation of $\operatorname{Hom}^{\prime \prime}(\mathbf{X}, \mathbf{Y})$. 
The first step is to determine the projectives of $\mathscr{F}$.

Proposition 3.4. If $\mathbf{X}$ is a filtered module then the following are equivalent:

(1) $X^{\prime \prime}$ is projective;

(2) the map $\tilde{h}$ of 3.3 is, for all $\mathbf{Y}$, an epimorphism;

(3) $\mathbf{X}$ is projective.

We shall prove $(1) \Rightarrow(2) ;(2) \Rightarrow(1) ;(1,2) \Rightarrow(3) ;(3) \Rightarrow(1)$.

Suppose first that $X^{\prime \prime}$ is projective. Thus $\mathbf{X}$ splits; let $\xi \in \Psi \mathbf{X}$. If $f^{\prime \prime}: X^{\prime \prime} \rightarrow Y^{\prime \prime}$ there is a $g: X^{\prime \prime} \rightarrow Y$ with $y^{\prime \prime} g=f^{\prime \prime}$. Set $f: \sum_{n=0}^{\infty} y^{n} g x^{\prime \prime} \xi^{n}$. Then $\left(f, f^{\prime \prime}\right): \mathbf{X} \rightarrow \mathbf{Y}$.

Now suppose $\mathbf{X}$ has the property (2). Define $\tilde{\mathbf{X}}=\left(0 \rightarrow \tilde{X} \rightarrow \tilde{X} \rightarrow X^{\prime \prime} \rightarrow 0\right)$ by $\tilde{X}_{q}=\sum_{n=-\infty}^{q} X_{n}^{\prime \prime}$, the maps in $\tilde{\mathbf{X}}$ being injections and projections. Then there is a map $\left(f, 1: X^{\prime \prime}\right): \mathbf{X} \rightarrow \tilde{\mathbf{X}}$ which by 2.1 is an isomorphism. Thus $\mathbf{X}$ splits; let $\xi \in \Psi X$. For any $q$ let $0 \rightarrow Y_{q-1} \rightarrow Y_{q} \rightarrow X_{q}^{\prime \prime} \rightarrow 0$ be exact and define a filtered module $Y$ by setting $Y_{r}=0, r<q-1$ and $Y_{r}=Y_{q}, r>q$. The associated graded module of $\mathbf{Y}$ is $X_{q}^{\prime \prime}$ in degree $q, Y_{q-1}$ in degree $q-1$ and 0 elsewhere. If $g^{\prime \prime}$ is the projection of $X^{\prime \prime}$ on $X_{q}^{\prime \prime}$ there is a map $\left(g, g^{\prime \prime}\right): \mathbf{X} \rightarrow \mathbf{Y}$, and $g_{q} \xi_{q}^{*}: X_{q} \rightarrow Y_{q}$ satisfies $y_{q}^{\prime \prime} g_{q} \xi_{q}^{*}=1: X_{q}^{\prime \prime}$. Thus for each $q, X_{q}^{\prime \prime}$ is projective, i.e., $X^{\prime \prime}$ is projective.

Now suppose $\mathbf{Y} \rightarrow \mathbf{Z}$ is a proper epimorphism. If $\mathbf{X}$ satisfies (1) and (2) then $\operatorname{Hom}\left(X^{\prime \prime}, Y^{\prime \prime}\right) \rightarrow \operatorname{Hom}\left(X^{\prime \prime}, Z^{\prime \prime}\right)$ is an epimorphism. But also, 3.3 is a filtered group in each case. It follows from 2.1 that $\operatorname{Hom}(\mathbf{X}, \mathbf{Y}) \rightarrow \operatorname{Hom}(\mathbf{X}, \mathbf{Z})$ is an epimorphism, so that $\mathbf{X}$ is projective.

Finally, if $\mathbf{X}$ is projective, let $f: Y^{\prime \prime} \rightarrow X^{\prime \prime}$ be an isomorphism with $Y^{\prime \prime}$ projective. Define $\mathbf{Y}$ by setting $Y_{q}=\sum_{n=-\infty}^{q} Y_{q}^{\prime \prime}$. Then there is a map $\left(f, f^{\prime \prime}\right): \mathbf{Y} \rightarrow \mathbf{X}$ which by 2.1 is a proper epimorphism. Since $\mathbf{X}$ is projective this splits, so that $X^{\prime \prime}$ is a direct summand of $Y^{\prime \prime}$.

The last step in the foregoing proof shows that for any $\mathrm{A} \in \mathscr{F}$ there is a projective $\mathbf{X}$ with a proper epimorphism $\mathbf{X} \rightarrow \mathbf{A}$. Indeed $\mathbf{X}$ may clearly be taken such that $X_{q}^{\prime \prime}=0$ whenever $A_{q}^{\prime \prime}=0$. It is obviously in the interest of economy to select $\mathbf{X}$ in this way; we shall in general assume the condition to hold without explicit mention.

4. Technical details; exact couples. We shall want to discuss exact couples

$$
C \stackrel{h}{\rightleftarrows} C
$$

of graded modules and, eventually, of multigraded modules. Suppose indeed that the modules are graded by an arbitrary abelian group and that $f, g, h$ are of degrees $\phi, \gamma, \chi$. We recall that such a couple has a sequence of derived couples

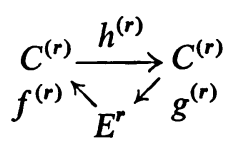


where $C^{(r)}=\operatorname{im} h^{r-1} \subset C$ and $\left\{E^{r}, d^{r}=g^{(r)} f^{(r)}\right\}$ is a spectral sequence; we have chosen our numbering so that $C^{\prime}=C, E^{1}=A$. The degrees of $f^{(r)}, g^{(r)}, h^{(r)}$ are, respectively, $\phi, \gamma+(1-r) \chi, \chi$ so that $\operatorname{deg} d^{r}=\phi+\gamma+(1-r) \chi$.

Now let us return for the moment to the case of simply graded modules. We shall want to study the effect of a certain finiteness condition. In particular we suppose $\operatorname{deg} f=-1, \operatorname{deg} g=0, \operatorname{deg} h=1$. Thus $\operatorname{deg} f^{(r)}=-1, \operatorname{deg} g^{(r)}=1-r$, $\operatorname{deg} h^{(r)}=1$.

Finiteness CONDITION 4.2. We suppose that for some $m$ and all $k<m, C_{k}=0$, and that for some $n$ and all $k>n, h_{k}: C_{k} \approx C_{k+1}$.

Observe first that this implies that $E^{1}=A$ is zero except in a finite number of degrees. Since $\operatorname{deg} d^{r}=-r$ this means that the spectral sequence is strongly convergent: $E^{k} \approx E^{k+1} \approx \cdots \approx E^{\infty}$ for some $k$. We propose to evaluate $E^{\infty}$ in this case. To this end let $n$ be sufficiently large so that $h_{k}: C_{k} \approx C_{k+1}$ for $k \geqq n$; we may identify all of these modules as $C_{\infty}$. We introduce a filtration $\boldsymbol{\Phi}_{h}=\left(0 \rightarrow \Gamma \rightarrow \Gamma \rightarrow \Gamma^{\prime \prime} \rightarrow 0\right)$ of $C_{\infty}$ by setting $\Gamma_{q}=\operatorname{im}\left[h^{n-q}: C_{q} \rightarrow C_{n}\right]$, the maps in $\Phi_{h}$ being the inclusions $\Gamma_{q-1} \subset \Gamma_{q}$ and the canonical maps onto the quotients $\Gamma_{q}^{\prime \prime}=\Gamma_{q} / \Gamma_{q-1}$. This filtration is clearly independent of $n$.

Proposition 4.3. $E^{\infty}$ is canonically isomorphic to $\Gamma^{\prime \prime}$.

Recalling that $\operatorname{im}\left(h^{r}\right)_{q} \subset C_{r+q}$ is the term of degree $r+q$ in $C^{(r+1)}$ we have, in the $(n-q+1)$ th derived couple, the exact sequence

$$
E_{n}^{n-q+1} \rightarrow \operatorname{im}\left(h^{n-q}\right)_{q-1} \rightarrow \operatorname{im}\left(h^{n-q}\right)_{q} \rightarrow E_{q}^{n-q+1} \rightarrow \operatorname{im}\left(h^{n-q}\right)_{2 q-n-1} .
$$

For large $n$ the last term is certainly 0 . But

$$
\operatorname{im}\left(h^{n-q}\right)_{q}=\Gamma_{q} \text { and } \operatorname{im}\left(h^{n-q}\right)_{q-1} \approx \operatorname{im}\left(h^{n-q+1}\right)_{q-1}=\Gamma_{q-1} .
$$

We may immediately generalize to multigraded modules, provided that the finiteness condition holds in the relevant degree uniformly in the others.

5. The spectral sequence for $\operatorname{Ext}(\mathbf{X}, \mathbf{Y})$. We recall first that if $A, B$ are objects of an abelian category and $X, Y$ are resolutions of $A, B$ then the graded group $\operatorname{Hom}(X, Y)$ is provided with a derivation $\delta f=(\partial f-1)^{\operatorname{deg} f} f \partial$ which makes it a chain complex. The cycles are chain maps from $X$ to $Y$, the boundaries are nullhomotopic maps and the homology consists of homotopy classes of maps. We have in fact $H_{-q} \operatorname{Hom}(X, Y)=\operatorname{Ext}^{q}(A, B)$ (cf. for example [1]).

Now suppose $\mathbf{A}$ and $\mathbf{B}$ are filtered modules and let $\mathbf{X}, \mathbf{Y}$ be projective resolutions of $\mathbf{A}$ and B. By Proposition 3.4 we have an exact sequence

$$
0 \rightarrow \operatorname{Hom}(\mathbf{X}, \mathbf{Y}) \stackrel{h}{\rightarrow} \operatorname{Hom}(\mathbf{X}, \mathbf{Y}) \stackrel{h^{\prime \prime}}{\rightarrow} \operatorname{Hom}\left(X^{n}, Y^{\prime \prime}\right) \rightarrow 0
$$

of chain complexes whose homology triangle is an exact couple

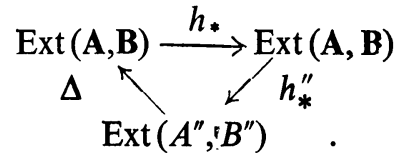


We shall adopt the convention that the terms $\operatorname{Ext}_{p}^{q}$ are of bidegree $(p,-q)$. Thus $\operatorname{deg} h_{*}=(1,0), \operatorname{deg} h_{*}^{\prime \prime}=(0,0)$ and $\operatorname{deg} \Delta=(-1,-1)$. We may refer to the degrees as filtering and resolvent degrees.

Written out, this exact couple becomes a family of exact sequences

$$
\cdots \rightarrow \operatorname{Ext}_{p}^{q}(\mathbf{A}, \mathbf{B}) \rightarrow \operatorname{Ext}_{p+1}^{q}(\mathbf{A}, \mathbf{B}) \rightarrow \operatorname{Ext}_{p+1}^{q}\left(A^{\prime \prime}, B^{\prime \prime}\right) \rightarrow \operatorname{Ext}_{p}^{q+1}(\mathbf{A}, \mathbf{B}) \rightarrow \cdots .
$$

It is not hard to see that these generalize the exact sequence of [1, Theorem 14.3].

Because of the finiteness of the filtrations involved the Finiteness Condition 4.2 holds in 5.1 for the filtering degree uniformly in the resolvent degree. We have thus, from 4.3, the following result.

THEOREM 5.2. The spectral sequence $E$ associated with the exact couple 5.1 has $E^{1}=\operatorname{Ext}\left(A^{\prime \prime}, B^{\prime \prime}\right)$. The term $E^{\infty}$ is the bigraded module associated with the filtration $\boldsymbol{\Phi}_{h_{*}}$ of $\operatorname{Ext}(\mathbf{A}, \mathbf{B})$.

We have departed here from the usual conventions for degrees in a spectral sequence; the bidegree of $d^{r}$ is $(-r,-1)$. It is convenient to represent $E^{\mathbf{1}}$ as follows:

$$
\begin{array}{lllll}
0 & \operatorname{Hom}_{1}\left(A^{\prime \prime}, B^{\prime \prime}\right) & \operatorname{Ext}_{1}^{1}\left(A^{\prime \prime}, B^{\prime \prime}\right) & \ldots & \uparrow \text { filtering degree } \\
0 & \operatorname{Hom}_{0}\left(A^{\prime \prime}, B^{\prime \prime}\right) & \operatorname{Ext}_{0}^{1}\left(A^{\prime \prime}, B^{\prime \prime}\right) & \ldots & \\
0 & \operatorname{Hom}_{-1}\left(A^{\prime \prime}, B^{\prime \prime}\right) & \operatorname{Ext}_{-1}^{1}\left(A^{\prime \prime} B^{\prime \prime}\right) & \ldots & \stackrel{\text { resolvent degree }}{\longleftarrow} .
\end{array}
$$

Thus $d^{r}$ goes 1 column to the right and $r$ rows down. Observe that all but finitely many rows are 0 .

We may take this occasion to point out that Theorem 5.2 provides an answer to a question asked above:

$$
\operatorname{Hom}_{p}^{\prime \prime}(\mathbf{A}, \mathbf{B}) \approx E_{p 0}^{\infty} .
$$

It is of course clear that the exact couple 5.1 is a contravariant functor of $\mathbf{A}$ and a covariant functor of $\mathbf{B}$. It follows immediately that if these are replaced by equivalent extensions of $A^{\prime \prime}$ and $B^{\prime \prime}$ then the corresponding exact couple may be mapped isomorphically onto 5.1 with the identity map connecting the bottom vertices. We have accordingly the following result.

THEOREM 5.4. The spectral sequence $E$ associated with 5.1 depends only on $A^{\prime \prime}, B^{\prime \prime}$ and the extension classes e A, e $\mathbf{B}$ of $\mathbf{A}$ and $\mathbf{B}$.

For $\mathfrak{a} \in \mathfrak{E} A^{\prime \prime}, \mathfrak{b} \in \mathfrak{E} B^{\prime \prime}$, then, we have a well-defined spectral sequence $E\left(A^{\prime \prime}, \mathfrak{a} ; B^{\prime \prime}, \mathfrak{b}\right)$; the sequence associated with 5.1 is $E\left(A^{\prime \prime}, \mathfrak{e A} ; B^{\prime \prime}, \mathrm{eB}\right)$.

6. The Postnikov invariant and $d^{1}$. We shall not investigate completely here the structure of the set $\mathbb{E} A^{\prime \prime}$ of extensions of a graded module $A^{\prime \prime}$ (but cf. [3] for 
a special case); we shall however want a "first approximation" which we shall now describe.

Let $\mathbf{A}$ be a filtered module. We consider the diagram

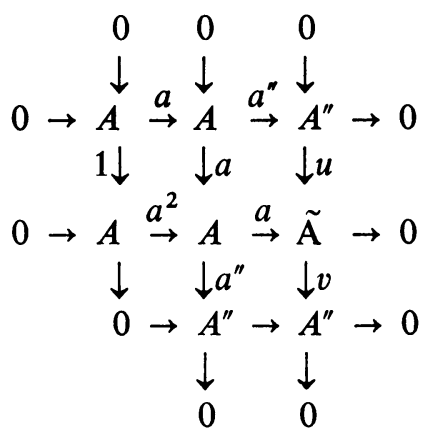

which commutes and has exact rows and columns, the first row and second column being $\mathbf{A}$, and $\tilde{A}$ being the cokernel of $a^{2}$. The degrees of $u$ and $v$ are, respectively, 1 and 0 . Thus the last column determines an extension class in $\operatorname{Ext}_{-1}^{1}\left(A^{\prime \prime}, A^{\prime \prime}\right)$. This obviously depends only on the extension class $\mathrm{eA} \in \mathbb{E} A^{\prime \prime}$; we have thus defined a map $\mathbf{p}: \mathfrak{E}^{\prime \prime} \rightarrow \operatorname{Ext}_{-1}^{1}\left(A^{\prime \prime}, A^{\prime \prime}\right)$. If $\mathfrak{a} \in \mathbb{E} A^{\prime \prime}$ (or $\mathbf{A}$ is a filtration with associated graded module $A^{\prime \prime}$ ) we refer to pa (or peA) as the Postnikov invariant of $\mathfrak{a}$ (or $\mathbf{A})$.

We may alternatively construct $\mathbf{p e A}$ as follows. Let $\mathbf{X}$ be a projective resolution of $\mathbf{A}$. Since $X^{\prime \prime}$ is projective $\mathbf{X}$ splits as a filtered graded module; say $\xi \in \Psi \mathbf{X}$. Notice that $\xi^{*}$ is of bidegree $(0,0)$. Then $x^{\prime \prime}\left(\partial \xi^{*}-\xi^{*} \partial\right)=0$ so that $\partial \xi^{*}-\xi^{*} \partial=x \hat{\xi}$ where $\hat{\xi} \in \operatorname{Hom}_{-1-1}\left(X^{\prime \prime}, X\right)$ is a chain map. Define

$$
\mathbf{o} \xi=x^{\prime \prime} \hat{\xi}=x^{\prime \prime} \xi\left(\partial \xi^{*}-\xi^{*} \partial\right)=x^{\prime \prime} \xi \partial \xi^{*} \text {. }
$$

This is a chain map in $\mathrm{Hom}_{-1-1}\left(X^{\prime \prime}, X^{\prime \prime}\right)$ and thus determines an element of $\operatorname{Ext}_{-1}^{1}\left(A^{\prime \prime}, A^{\prime \prime}\right)$.

Proposition 6.2. If $\xi \in \Psi \mathbf{X}$ then the class of $\mathbf{o} \xi=x^{\prime \prime} \xi \partial \xi^{*}$ is peA.

This is easily seen by taking a "projective resolution" of 6.1 , i.e., by replacing $A$ by $X, a$ by $x, \cdots$, throughout.

Using these Postnikov invariants it is possible to give the following simple description of $d^{1}$ in the spectral sequence introduced above.

THEOREM 6.3. In the spectral sequence $E\left(A^{\prime \prime}, \mathfrak{a} ; B^{\prime \prime}, \mathfrak{b}\right)$ the map $d_{* q}$ : $\operatorname{Ext}^{q}\left(A^{\prime \prime} B^{\prime \prime}\right) \rightarrow \operatorname{Ext}^{q+1}\left(A^{\prime \prime} B^{\prime \prime}\right)$ is given by

$$
d^{1} \phi=(\mathbf{p b}) \phi-(-1)^{q} \phi(\mathbf{p a}) .
$$

Let $\mathbf{X}, \mathbf{Y}$ be projective resolutions of $\mathbf{A}, \mathbf{B}$, where $e \mathbf{A}=\mathfrak{a}, e \mathbf{B}=\mathfrak{b}$, and suppose $\xi \in \Psi \mathbf{X}, \eta \in \Psi \mathbf{Y}$. Suppose the chain map $f^{\prime \prime} \in \operatorname{Hom}_{p, q}\left(X^{\prime \prime}, Y^{\prime \prime}\right)$ determines the homotopy class $\phi=\left[f^{\prime \prime}\right] \in \operatorname{Ext}_{p}^{-q}\left(A^{\prime \prime}, B^{\prime \prime}\right)$; we must evaluate $d^{1}\left[f^{\prime \prime}\right]=h_{*}^{\prime \prime} \Delta\left[f^{\prime \prime}\right]$. 
We may suppose that $\left(f, f^{\prime \prime}\right): \mathbf{X} \rightarrow \boldsymbol{Y}$. Then $h^{\prime \prime} \delta\left(f, f^{\prime \prime}\right)=0$, so that

$$
\delta\left(f, f^{\prime \prime}\right)=\left(\delta f, \delta f^{\prime \prime}\right)=(y g, 0)
$$

where $\left(g, g^{\prime \prime}\right): \mathbf{X} \rightarrow \mathbf{Y}$ is in the class $\Delta\left[f^{\prime \prime}\right]$. But then $\left[g^{\prime \prime}\right]=d 1\left[f^{\prime \prime}\right]$.

On the other hand we have $g=\eta \delta f, g^{\prime \prime}=y^{\prime \prime} \eta \delta f \xi^{*}$ so that, using 3.2,

$$
\begin{aligned}
g^{\prime \prime}= & y^{\prime \prime} \eta \partial \eta^{*} y^{\prime \prime} f \xi^{*}+y^{\prime \prime} \eta \partial y \eta^{*} y^{\prime \prime} \eta f \xi^{*} \\
& -(-1)^{q}\left\{y^{\prime \prime} \eta y \eta^{*} y^{\prime \prime} \eta f \xi^{*} x^{\prime \prime} \partial \xi^{*}+y^{\prime \prime} \eta y \eta^{*} y^{\prime \prime} \eta f x \xi^{*} x^{\prime \prime} \xi \partial \xi^{*}\right\},
\end{aligned}
$$

the other terms clearly vanishing. Thus, recalling that $y^{\prime \prime} f \xi^{*}=f^{\prime \prime}$

$$
g^{\prime \prime}=(\mathbf{o} \eta) f^{\prime \prime}-(-1)^{q} f^{\prime \prime}(\mathbf{o} \xi)+\delta\left(y^{\prime \prime} \eta f \xi^{*}\right)
$$

so that

$$
d^{1}\left[f^{\prime \prime}\right]=[\mathbf{o} \eta]\left[f^{\prime \prime}\right]-(-1)^{q}\left[f^{\prime \prime}\right][\mathbf{o} \xi]=(\mathbf{p} \mathfrak{b}) \phi-(-1)^{q} \phi(\mathbf{p a}) .
$$

7. The triple product and $d^{2}$. We shall confine our attention here to short filtrations, i.e., filtrations $\mathbf{A}$ whose associated graded modules are nonzero in two degrees only: we may take these to be 0 and 1 . This is of course a very great restriction; such an $\mathbf{A}$ is completely determined by the short exact sequence $0 \rightarrow A_{0}^{\prime \prime} \rightarrow A_{1} \rightarrow A_{1}^{\prime \prime} \rightarrow 0$. The extension class of the sequence is just (peA) $)_{1} \in$ $\operatorname{Ext}^{1}\left(A_{1}^{\prime \prime}, A_{0}^{\prime \prime}\right)$, and indeed $\mathfrak{E} A^{\prime \prime}$ becomes identified in this way with this group.

For A,B such filtrations we propose to compute $d^{2}$ in the spectral sequence $E\left(A^{\prime \prime}, \mathrm{eA} ; B^{\prime \prime}, \mathrm{eB}\right)$. Since the $d^{r}, r>2$, are all zero this completes the computation of the spectral sequence and in particular of $\operatorname{Hom}^{\prime \prime}(\mathbf{A}, \mathbf{B})$, which is associated to a filtration of $\operatorname{Hom}\left(A_{\infty}, B_{\infty}\right)=\operatorname{Hom}\left(A_{1}, B_{1}\right)$. In other words we shall have computed, up to extensions, the group of homomorphisms of the middle terms of two short exact sequences, in terms of the ends and the extension classes.

The computation of $d^{2}$ will be given in terms of a secondary operation reminiscent of the Massey triple product.

Let us consider bigraded modules $X$ with $X_{i j}=0$ for $i \neq 0,1$, provided with derivations of degree $(0,-1)$. If $X, Y$ are such objects we write $\operatorname{Hom}^{b}(X, Y)$ for bigraded group of homotopy classes of chain maps $X \rightarrow Y$. If $\alpha \in \operatorname{Hom}_{-1,-1}^{b}(X, X)$ and $\beta \in \operatorname{Hom}_{-1,-1}^{b}(Y, Y)$ we define a primary operation $\nabla_{1}(\alpha, \beta): \operatorname{Hom}^{b}(X, Y)$ $\rightarrow \operatorname{Hom}^{b}(X, Y)$, of degree $(-1,-1)$, by setting

$$
\nabla_{1}(\alpha, \beta) \phi=\beta \phi-(-1)^{q} \phi \alpha
$$

where $q$ is the "resolvent" degree of $\phi$, i.e., $\phi \in \operatorname{Hom}_{* q}^{b}(X, Y)$. The secondary operation is $\nabla_{2}(\alpha, \beta): \operatorname{Ker} \nabla_{1}(\alpha, \beta) \rightarrow \operatorname{Coker} \nabla_{1}(\alpha, \beta)$. It is of degree $(-2,-1)$, and is defined as follows.

Let $a, b$ be maps in the homotopy classes $\alpha, \beta$. Since they are of degree $(-1,-1)$ their squares are 0 . Now suppose $f \in \operatorname{Hom}_{p, q}(X, Y)$ has its homotopy class $[f]$ in the kernel of $\nabla_{1}(\alpha, \beta)$. Then $b f-(-1)^{q} f a=\delta g$ for some $g \in \operatorname{Hom}_{p-1, q}(X, Y)$. for such a $g$ 


$$
\left(b g-(-1)^{q} g a\right)=-b \delta g-(-1)^{q}(\delta g) a=0
$$

so that $(-1)^{q} g a-b g$ determines an element of $\operatorname{Hom}_{p-2, q-1}^{b}(X, Y)$. The image of this in the cokernel of $\nabla_{1}(\alpha, \beta)$ is $\nabla_{2}(\alpha, \beta)[f]$; it is easily verified that this is independent of the choices of $a, b, f, g$.

We may in particular apply this construction in the case that $X, Y$ are projective resolutions of graded modules $A, B$ which are nonzero in degrees 0 and 1 only. If $\alpha \in \operatorname{Ext}_{-1}^{1}(A, A)$ and $\beta \in \operatorname{Ext}_{-1}^{1}(B, B)$ then $\nabla_{1}(\alpha, \beta): \operatorname{Ext}(A, B) \rightarrow \operatorname{Ext}(A, B)$ and $\nabla_{2}(\alpha, \beta)$ is thus a secondary operation on $\operatorname{Ext}(A, B)$.

THEOREM 7.1. Let $\mathbf{A}$ and $\mathbf{B}$ be short filtrations and su ppose $p: Z\left(E^{1}\right) \rightarrow E^{2}$ is the canonical epimorphism in the spectral sequence $E\left(A^{\prime \prime}, \mathrm{eA} ; B^{\prime \prime}, \mathrm{eB}\right)$. Then $d^{1}=\nabla_{1}\left((\mathbf{p e A})_{1},(\mathbf{p e B})_{1}\right)$ and $d^{2} p=\nabla_{2}\left((\mathbf{p e A})_{1},(\mathbf{p e B})_{1}\right)$.

We take, as above, projective resolutions $\mathbf{X}$ of $\mathbf{A}$ and $\mathbf{Y}$ of $\mathbf{B}$. Suppose $\xi \in \Psi \mathbf{X}, \eta \in \Psi \mathbf{Y}$ so that $\boldsymbol{o} \xi \in \mathbf{p e A}$ and $\boldsymbol{o} \eta \in \mathbf{p e B}$. An element of $Z\left(E^{\mathbf{1}}\right)$ is represented by the homotopy class $\left[f^{\prime \prime}\right]$ of some $f^{\prime \prime} \in \operatorname{Hom}_{p, q}\left(X^{\prime \prime}, Y^{\prime \prime}\right)$, and we may suppose that $\left(f, f^{\prime \prime}\right): \mathbf{X} \rightarrow \mathbf{Y}$. Since $d^{1}\left[f^{\prime \prime}\right]=0$ we have

$$
\begin{aligned}
\delta\left(f, f^{\prime \prime}\right) & =h^{2}\left(g, g^{\prime \prime}\right)+h \delta\left(w, w^{\prime \prime}\right) \\
& =\left(y^{2} g, 0\right)+(y \delta w, 0)
\end{aligned}
$$

where $\left(g, g^{\prime \prime}\right) \in \operatorname{Hom}_{p-2, q-1}(\mathbf{X}, \mathbf{Y})$ and $\left(w, w^{\prime \prime}\right) \in \operatorname{Hom}_{p-1, q}(\mathbf{X}, \mathbf{Y})$. But then $\left(f-y w, f^{\prime \prime}\right) \in \operatorname{Hom}_{p, q}(\mathbf{X}, \mathbf{Y})$, so that we may assume that $\delta\left(f, f^{\prime \prime}\right)=\left(y^{2} g, 0\right)$. But then $d^{2} p\left[f^{\prime \prime}\right] \ni\left[g^{\prime \prime}\right]$. On the other hand, $g=\eta^{2} \delta f$ so that, using 3.2,

$$
\begin{aligned}
& g^{\prime \prime}= y^{\prime \prime} \eta^{2}(\delta f) \xi^{*}=y^{\prime \prime} \eta^{2} \partial f \xi^{*}-(-1)^{q} y^{\prime \prime} \eta^{2} f \partial \xi^{*} \\
&=y^{\prime \prime} \eta^{2} \partial \eta^{*} y^{\prime \prime} f \xi^{*} x^{\prime \prime} \xi^{*}+y^{\prime \prime} \eta \partial \eta^{*} y^{\prime \prime} \eta f \xi^{*} x^{\prime \prime} \xi^{*}+y^{\prime \prime} \partial \eta^{*} y^{\prime \prime} \eta^{2} f \xi^{*} x^{\prime \prime} \xi^{*} \\
&-(-1)^{q}\left\{y^{\prime \prime} \eta^{*} y^{\prime \prime} \eta^{2} f \xi^{*} x^{\prime \prime} \partial \xi^{*}+y^{\prime \prime} \eta^{*} y^{\prime \prime} \eta^{2} f x \xi^{*} x^{\prime \prime} \xi \partial \xi^{*}\right. \\
&\left.+y^{\prime \prime} \eta^{*} y^{\prime \prime} \eta^{2} f x^{2} \xi^{*} x^{\prime \prime} \xi^{2} \partial \xi^{*}\right\}
\end{aligned}
$$

the other terms all clearly vanishing. We may further evaluate this as follows:

$$
g^{\prime \prime}=(\mathbf{o} \eta)\left(y^{\prime \prime} \eta f \xi^{*}\right)-(-1)^{q}\left(y^{\prime \prime} \eta f \xi^{*}\right)(\mathbf{o} \xi)+\delta\left(y^{\prime \prime} \eta^{2} f \xi^{*}\right)
$$

since $y^{\prime \prime} \eta^{2} \partial y^{*}$ and $x^{\prime \prime} \xi^{2} \partial \xi^{*}$, being of filtering degree -2 both vanish. Thus

$$
\left[g^{\prime \prime}\right]=\left[(\mathbf{o} \eta)\left(y^{\prime \prime} \eta f \xi^{*}\right)-(-1)^{q}\left(y^{\prime \prime} \eta f \xi^{*}\right)(\mathbf{o} \xi)\right] \text {. }
$$

It remains only to evaluate $\delta\left(y^{\prime \prime} \eta \xi^{*}\right)$; referring to 6.4 we see it is $-\left\{(0 \eta) f^{\prime \prime}-(-1)^{q} f^{\prime \prime}(\mathbf{o} \xi)\right\}$.

\section{BIBLIOGRAPHY}

1. A. Heller, Homological algebra in abelian categories, Ann. of Math. (2) 68 (1958), 484-525.

2. - On the Künneth theorem, Trans. Amer. Math. Soc. 98 (1961), 450-458.

3. —_ On the classification of filtered modules, Illinois J. Math. 5 (1961), 412-419.

UNIVERSITY OF ILLINOIS,

URBANA, ILLINOIS 